\title{
Aplicación de biomateriales de base biológica, moléculas bioactivas e ingeniería de tejidos en cirugía plástica periodontal. Una revisión
}

\section{Application of biological based biomaterials, bioactive molecules and tissue engineering in periodontal plastic surgery. A review}

\author{
Durán Yaneth JC ${ }^{1}$, Alarcón $C^{2}$, Velásquez $D^{3}$
}

\begin{abstract}
RESUMEN
Objetivo: Describir, clasificar y discutir las indicaciones de los biomateriales de base biológica, moléculas bioactivas e ingeniería de tejidos que se están usando para el manejo de recesiones y aumento de encía en cirugía plástica periodontal. En esta revisión de la literatura, se utilizó una combinación de los términos de búsqueda específicos que consideraran los materiales en revisión, para el aumento de encía adherida, y el recubrimiento radicular. Materiales y Métodos: Se usaron las siguientes fuentes: Medline, Biblioteca Cochrane, y búsqueda manual de revistas específicas como el Journal of Periodontology, International Journal of Periodontics and Restorative Dentistry y Journal of Clinical Periodontology entre años 1985 y 2011. Se revisaron un total de 117 artículos y se seleccionaron 74 entre estudios clínicos controlados, estudios clínicos randomizados, reportes de casos y estudios en animales. Los artículos fueron revisados por los autores y aceptados por consenso para su discusión. Conclusiones: 1) Existe una serie de materiales que presentan gran potencial y podrían ser una alternativa viable a los injertos autógenos, pero se requiere más estudios a largo plazo. 2) Existe necesidad de estudios con la investigación de estos procedimientos en relación a resultados orientados a la estabilidad, seguridad y efectividad de los diferentes materiales existentes.
\end{abstract}

Rev. Clin. Periodoncia Implantol. Rehabil. Oral Vol. 5(3); 144-151, 2012.

Palabras clave: Recesión gingival, cirugía mucogingival, injerto de tejido, dispositivo biológico, moléculas bioactivas, dispositivos de células vivas.

\begin{abstract}
Objective: To describe, classify and discuss the clinical applications of biologically based biomaterials, bioactive molecules and tissue engineering being utilized in gingival recession therapy and gingival augmentation procedures in plastic periodontal surgery. In this literature review, a combination of specific search key words were used, including materials being reviewed, indicated for gingival augmentation and root coverage procedures. Materials and Methods: The following sources were consulted: Medline, Cochrane Library and manual search of specific scientific journals such as Journal of Periodontology, International Journal of Periodontics and Restorative Dentistry and Journal of Clinical Periodontology between the years 1985 and 2011. A total of 117 articles were reviewed with 74 being selected unanimously by the authors for discussion in the manuscript. These articles included controlled clinical studies, randomized clinical studies, case reports and animal studies. The selected articles were reviewed by the authors and accepted by consensus. Conclusions: 1) There is a cohort of materials that exhibit great potential which could be a viable alternative to autografts but are in need of further long term studies. 2) There is a need of research of these materials in relation to stability, safety and efficacy.
\end{abstract}

Rev. Clin. Periodoncia Implantol. Rehabil. Oral Vol. 5(3); 144-151, 2012.

Key words: Gingival recession, mucogingival surgery, tissue graft, biologic dispositives, bioactive molecules, life cell constructs.

\section{INTRODUCCIÓN}

El aumento de encía adherida y el recubrimiento de superficies radiculares han sido objeto de innumerables esfuerzos y proyectos de investigación guiados al establecimiento y selección de técnicas quirúrgicas y materiales que permitan lograr de una manera predecible, y no invasiva, el restablecimiento de tejidos periodontales compatibles con salud, función y estética periodontal.

En 1963 Björn ${ }^{(1)}$ describe por primera vez la técnica de injerto libre de encía, indicado inicialmente para el aumento de encía adherida. Posteriormente, a mediados de la década de los $80^{\prime}$ Langer y Langer(2), describen la utilización del injerto de tejido conectivo subepitelial para el manejo de las recesiones gingivales. Estas técnicas tienen como desventaja la disección del injerto desde un segundo lecho quirúrgico (aumentando así el tiempo de operación y consecuentemente el riesgo de morbilidad e infección) y una cantidad limitada de tejido en el sitio donante $^{(3)}$. En consecuencia, se han desarrollado alternativas para reemplazar la utilización de tejidos nativos.

1. Especialista en Periodoncia, Universidad Chile. Diplomado en Prótesis Fija, Universidad de Chile. Docente Clínica Integral, Universidad del Desarrollo. Práctica Privada. Santiago, Chile.

2. Especialista en Periodoncia, Universidad de Valparaíso. Especialista en Implantología Oral, Universidad Andrés Bello. Práctica Privada. Santiago, Chile.

3. DDS, MSD. Especialista en Prostodoncia y Materiales Dentales, Indiana University. Especialista en Periodoncia e Implantología, Universidad de Texas-San Antonio. Diplomate American Board of Periodontology. Profesor Adjunto Clínico, Michigan University School of Dentistry. Departamento de Periodoncia y Medicina Oral. Práctica Privada. Fenton, Michigan, USA. 
El objetivo de esta revisión es describir, clasificar y discutir las indicaciones de los biomateriales de base biológica, moléculas bioactivas e ingeniería de tejidos que se están usando para el manejo de recesiones y aumento de encía adherida en cirugía plástica periodontal.

\section{MATERIALES Y MÉTODOS}

Se usaron las siguientes fuentes: Medline, Biblioteca Cochrane, y búsqueda manual de revistas específicas como el Journal of Periodontology, International Journal of Periodontics and Restorative Dentistry y Journal of Clinical Periodontology entre años 1985 y 2011. Se utilizaron los siguientes términos de búsqueda: Recesión Gingival, Cirugía Mucogingival, Injerto de Tejido, Dispositivo Biológico, Moléculas Bioactivas, Dispositivos de Células Vivas utilizando nombres genéricos y comerciales como está ilustrado en las Tablas 1 y 2.

De un total de 117 artículos, se seleccionaron 74 para esta revisión incluyendo estudios clínicos controlados, estudios clínicos randomizados, reportes de casos y estudios en animales. Los artículos fueron revisados por los autores y aceptados por consenso para su discusión.

Tabla 1. Siglas y significado.

\begin{tabular}{|c|c|}
\hline Sigla & Significado \\
\hline (IGL) & Injerto gingival libre \\
\hline (ITCS) & Injerto tejido conectivo subepitelial \\
\hline (MDA) & Matriz dérmica acelular \\
\hline$(\mathrm{CDC})$ & Colgajo desplazado coronario \\
\hline (CDCT) & Colgajo desplazado coronario tunelización \\
\hline (MG) & Mucograft ${ }^{\circledR}$ \\
\hline (DMPE) & Derivados de matriz de proteínas del esmalte \\
\hline (FCDP) & Factores de crecimiento derivados de plaquetas \\
\hline (FCDF) & Factor de crecimieno derivado del fibroblasto \\
\hline (SDD-FH) & $\begin{array}{l}\text { Sustituto dérmico derivado de } \\
\text { fibroblastos humanos vivos }\end{array}$ \\
\hline (TCB) & Terapia celular bilaminar \\
\hline (PRP) & Plasma rico en plaquetas \\
\hline (ROG) & Regeneración ósea guiada \\
\hline (RTG) & Regeneración tisular guiada \\
\hline (DM) & Dynamatrix ${ }^{\circledR}$ \\
\hline (MCV) de sus siglas en Inglés: LCC & $\begin{array}{l}\text { Matriz de células vivas } \\
\text { Living cellular construct }\end{array}$ \\
\hline
\end{tabular}

\section{DISPOSITIVOS CON BASE BIOLÓGICA}

\section{A) DISPOSITIVOS ALOGRÁFICOS:}

Durante la década del 70' se probaron diferentes materiales alográficos tales como: el injerto de tejido preservado de esclera, la dura madre liofilizada, matriz dérmica congelada y disecada, con pobres resultados para el manejo de defectos mucogingivales ${ }^{(4)}$. Dentro de este grupo de dispositivos la Matriz Dérmica Acelular (MDA), es la que más interés ha creado desde el punto de vista clínico en el área de la cirugía plástica periodontal, siendo por esto una de las más estudiadas.
Tabla 2.

\begin{tabular}{|c|c|c|}
\hline Tipo de Material & $\begin{array}{l}\text { Nombre } \\
\text { Comercial }\end{array}$ & $\begin{array}{l}\text { Indicaciones } \\
\text { Terapéuticas }\end{array}$ \\
\hline $\begin{array}{l}\text { 1. BIOMATERIALES DE } \\
\text { BASE BIOLÓGICA }\end{array}$ & & \\
\hline Injertos alográficos & & \\
\hline - Matriz dérmica acelular & $\begin{array}{l}\text { Alloderm } \circledast \text {, } \\
\text { Puros dermis } ®\end{array}$ & $\begin{array}{l}\text { Recesiones, aumento } \\
\text { encía adherida }\end{array}$ \\
\hline Injertos xenográficos & & \\
\hline - Membranas colágenas & 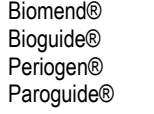 & $\begin{array}{l}\text { Recesiones, aumento } \\
\text { encía adherida }\end{array}$ \\
\hline $\begin{array}{l}\text { - Matriz de colágeno bicapa de } \\
\text { origen porcino }\end{array}$ & Mucograft $\circledast$ & $\begin{array}{l}\text { Recesiones, aumento } \\
\text { encía adherida }\end{array}$ \\
\hline $\begin{array}{l}\text { 2. BIOMATERIALES BASE MOLÉ- } \\
\text { CULAS BIOACTIVAS }\end{array}$ & & \\
\hline $\begin{array}{l}\text { - Membrana de matriz extracelular } \\
\text { derivada de submucosa del tejido del } \\
\text { intestino delgado de origen porcino } \\
\text { glicosaminglicanos }\end{array}$ & Dynamatrix@ & Aumento encía adherida. \\
\hline $\begin{array}{l}\text { - Factor de crecimiento derivado de } \\
\text { plaquetas }\end{array}$ & $\begin{array}{l}\text { Rh-FCDP-BB } \\
\text { GEM21@ }\end{array}$ & Recesiones \\
\hline $\begin{array}{l}\text { - Derivados de matriz de proteínas } \\
\text { del esmalte }\end{array}$ & Emdogain ${ }^{\circledR}$ & Recesiones \\
\hline - Peptidos de adhesión celular & P-15® & Recesiones \\
\hline $\begin{array}{l}\text { 3. BIOMATERIALES BASE CÉLULAS } \\
\text { VIVAS (INGENIERÍA TEJIDOS) }\end{array}$ & & \\
\hline $\begin{array}{l}\text { - Terapia fibloblastos autógenos } \\
\text { inyectados }\end{array}$ & & Regeneración de papila \\
\hline $\begin{array}{l}\text { - Sustitutos derivados de fibroblastos } \\
\text { humanos }\end{array}$ & Dermagraft $\circledast$ & $\begin{array}{l}\text { Recesiones, aumento } \\
\text { encia adherida }\end{array}$ \\
\hline - Plasma rico en plaquetas & & Recesiones \\
\hline $\begin{array}{l}\bullet \mathrm{TCB} \\
\bullet \mathrm{MCV}\end{array}$ & Celltex® & $\begin{array}{l}\text { Aumento de encía } \\
\text { adherida }\end{array}$ \\
\hline
\end{tabular}

Matriz Dérmica Acelular

La MDA se procesa a partir de la piel obtenida de los donantes de tejidos humanos almacenados en bancos certificados. Los tejidos del donante se preparan mediante la eliminación de la epidermis y los componentes celulares de la piel. La matriz de colágeno acelular es entonces crio-protegida y rápidamente liofilizada, esto para preservar su integridad estructural y bioquímica ${ }^{(5)}$. En periodoncia e implantología, se ha estudiado, principalmente, para el aumento de encía adherida y en el manejo de las recesiones gingivales (Figura 1). 


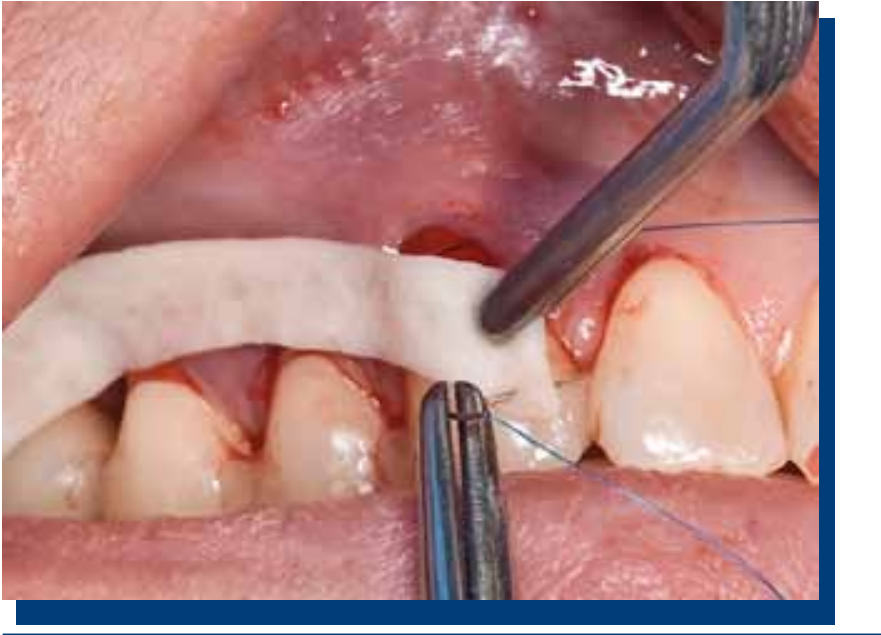

Figura 1. Matriz dérmica acelular.

\section{Matriz Dérmica Acelular en Aumento de Encía Adherida}

Wei y col. ${ }^{(1)}$, realizaron un estudio clínico donde compararon la MDA con injerto gingival libre (IGL), para el aumento de encía adherida en sitios con $\leq 1 \mathrm{~mm}$. A los 6 meses, encontraron como resultado que la MDA fue menos predecible que el IGL en términos de aumentar el tejido queratinizado, debido a la considerable contracción y la calidad desigual del tejido agregado ganado. Pero el resultado estético usando la MDA, fue superior que el grupo que usó IGL. Posteriormente, Harris( ${ }^{(6)}$ comparó la MDA, el IGL y el injerto de tejido conectivo en tres grupos de pacientes con $\leq 1 \mathrm{~mm}$ de encía adherida. A los 6 meses, encontró que el aumento de encía adherida fue estadísticamente significativo en los tres grupos estudiados, observando los mejores resultados con la MDA y el IGL con $4.1 \mathrm{~mm}$, versus $3.6 \mathrm{~mm}$ con el ITCS. Recientemente, Scarano y col. ${ }^{(7)}$ en un estudio clínico e histológico con 10 pacientes con $\leq 1 \mathrm{~mm}$ de encía adherida y tratados con MDA, concluyeron que a los 3 meses hubo una ganancia del ancho de encía adherida de $2.92 \mathrm{~mm}$ en promedio, y que a las 10 semanas la MDA fue completamente reepitelizada y sustituida en el tejido nativo en humanos.

\section{Estudios Histológicos de Matriz Dérmica Acelular}

Se ha demostrado la biocompatibilidad de este material y se ha concluido que la MDA no tiene mucha capacidad de inducir cito diferenciación ${ }^{(8)}$. Sin embargo, esto no sería relevante ya que esta propiedad se atribuye más al microambiente tejido conectivo/epitelio de la zona receptora. Otro reporte histológico de un caso en humanos ${ }^{(9)}$, observó que a los 4 meses, la MDA no se revasculariza completamente en su porción coronal, contrario a lo que reportaron otros investigadores que compararon la MDA con el ITCS, en humanos a los 6 meses $^{(10)}$ y en perros a las 12 semanas ${ }^{(11)}$. En estos últimos estudios, no se encontraron diferencias significativas en ninguno de los dos grupos estudiados, en cuanto a revascularización, presencia de colágeno y fibroblastos. La MDA se observó bien integrada dentro de una estructura altamente vascularizada, lo que indica la incorporación completa de la MDA al tejido nativo.

Matriz Dérmica Acelular vs. Injerto de Tejido Conectivo Subepitelial en el Tratamiento de Recesión Gingival

Algunos estudios clínicos randomizados ${ }^{(12,13)}$ han comparado la MDA con el ITCS para el tratamiento de la recesión gingival tipo I-II de Miller $\geq 2 \mathrm{~mm}$. Después de 6 meses de observación, concluyeron no encontrar diferencias significativas entre el grupo test (MDA) y el grupo control (ITCS), en términos de recubrimiento radicular, avalando así el uso de la MDA como un sustituto para el ITCS. Posteriormente, Tal y col. (14) compararon la MDA y el ITCS en el tratamiento de recesiones de $\geq 4$ $\mathrm{mm}$. En sus resultados reportaron que no había diferencias significativa a los 12 meses de estudio en cuanto a recubrimiento radicular, pero si hubo diferencias positivas en el aumento de tejido queratinizado en los resultados del grupo de ITCS. En el 2004, Harris ${ }^{(15)}$ publicó un estudio retrospectivo comparativo a corto (promedio 3 meses) y largo plazo (promedio 4 años) en recesiones tratadas con MDA e ITCS. Se concluyó que en cuanto a porcentaje de recubrimiento radicular, el corto plazo de la MDA y del ITCS fue de $93.4 \%$, y $96.6 \%$ respectivamente y el largo plazo del ITCS fue $97 \%$. Estos tres grupos fueron estadísticamente mayores a los encontrados en el largo plazo del MDA, que fue $65.8 \%$. Por lo tanto, el promedio de los resultados con ITCS mejoró con el tiempo, comparado con el promedio de los resultados con MDA, en los cuales solo el $32 \%$ de los casos tratados con MDA mejoraron o se mantuvieron estables a largo plazo. Estos resultados son avalados posteriormente por Hirsch y col. ${ }^{\left({ }^{16)}\right.}$, en su estudio comparativo de la MDA y del ITCS, en el que después de dos años concluye que los dos injertos estudiados son predecibles para el tratamiento de las recesiones gingivales y son estables longitudinalmente. Sin embargo, el ITCS tiene una mejoría significativa en recubrimiento radicular, en ganancia de encía adherida, en ganancia de inserción y en disminución de la profundidad al sondaje residual.

Matriz Dérmica Acelular (MDA) vs. Colgajo Desplazado Coronal (CDC) en el Tratamiento de Recesión Gingival

En otros estudios ${ }^{(17,18)}$, se ha comparado el CDC sólo y el CDC + MDA. A los 6 meses, no se observaron diferencias significativas en cuanto a recubrimiento radicular, pero sí se encontró un aumento significativo en el aumento de grosor de la encía en el grupo tratado con MDA. Otro estudio(19) para el tratamiento de recesiones Miller clase I-II, comparó la técnica convencional CDC + MDA y la técnica modificada CDC + MDA, que consistió en una extensión del colgajo. Se concluyó que tuvo mejores resultados estadísticamente significativos y clínicos la técnica modificada. Las técnicas convencionales quirúrgicas diseñadas para los injertos autógenos podrían no ser apropiadas cuando se usa injerto de MDA. Recientemente, Taylor y col. ${ }^{(20)}$, propusieron una técnica modificada sin tensión gingival usando MDA + CDC. Se describen cambios en la técnica de sutura del colgajo y de la MDA, modificaciones en el grosor de ésta y su adaptación al suturar contra la superficie radicular y el tejido óseo. Con estas variaciones, se logró mejorar la predictibilidad clínica y los resultados fueron comparables a aquellos obtenidos en otros reportes usando tejido autógeno.

\section{Matriz Dérmica Acelular y su Uso con Otras Técnicas Quirúrgicas} en el Tratamiento de Recesión Gingival

Un estudio clínico comparó el CDC, con (grupo control) y sin incisiones verticales (grupo experimental), en recesiones Miller clase I-II menor o igual a $2 \mathrm{~mm}^{(21)}$. Se concluyó que en el grupo en el que se hicieron incisiones verticales, se obtuvo mayor resultado estadísticamente significativo en porcentaje de recubrimiento radicular, en sitios con recesiones localizadas. A los 6 meses se encontró una reducción de la recesión gingival estadísticamente significativa favoreciendo al grupo control con un $84.1 \%$, en comparación con el experimental que obtuvo un $68.9 \%$. Otros autores han comparado la técnica de tunelización posicionada coronalmente (TPC) + MDA con CDC + $\mathrm{MDA}^{(22)}$. Estos encontraron que el CDC + MDA alcanzó un recubrimiento radicular de un $95 \%$, muy distante del $78 \%$ alcanzado por la técnica de TPC. Igualmente, midieron la predictibilidad de la técnica donde se alcanzó un $83 \%$ para el grupo CDC + MDA contra un 50\% para el grupo tratado con TPC + MDA. Sin embargo, los pacientes de este último grupo reportaron menores molestias postoperatorias.

Para el tratamiento de múltiples recesiones y zonas de compromiso estético, se han descrito las técnicas de tunelización + MDA, con pequeñas modificaciones, donde se han reportado 5 casos clínicos con $93.5 \%$ de recubrimiento radicular a los 12 meses $^{(23)}$, y en uno de los casos un $100 \%$ recubrimiento radicular a los 2 meses $^{(24)}$. Esto sugiere que el uso de la MDA con tunelización en múltiples recesiones puede ser una alternativa viable a la técnica tradicional con ITCS y, especialmente, se debe considerar en pacientes con limitaciones de tejido conectivo. Otro estudio $^{(25)}$ ha reportado la incorporación del plasma rico en plaquetas (PRP) al MDA, combinando con la técnica de tunelización. En estos se encontró un porcentaje de un $90 \%$ de recubrimiento radicular cuando se adiciona esta membrana biológica, versus un $70 \%$ de recubrimiento cuando no se usa.

En una revisión sistemática relacionada con la terapia de MDA, se concluye que esta técnica tiene gran potencial en la reparación de recesión gingival y para aumento de encía y tejido queratinizado. A pesar, de las tendencias encontradas, existen diferencias en los diseños de los estudios y falta de datos estandarizados que impiden llegar a conclusiones definitivas y análisis más comprensivos ${ }^{(5)}$. 


\section{B) DISPOSITIVOS XENOGRÁFICOS:}

\section{Membranas Colágenas}

Los biomateriales xenográficos y, especialmente, las membranas colágenas (de origen porcino y bovino) han sido ampliamente utilizadas en medicina y odontología. Fueron inicialmente pensadas como barrera en regeneración ósea guiada (ROG) y en regeneración tisular guiada (RTG), ya que su característica reabsorbible evitaba una segunda cirugía. Son fabricadas con colágeno tipo I, ya que este es el mayor componente del tejido conectivo humano. También encontramos algunas de colágeno tipo III, sulfato 4 de condroitina, atelo colágeno y tendón de colágeno(26). En la mayoría de estudios clínicos comparativos de ITCS + CDC versus el uso de CDC + membranas colágenas en el tratamiento para recesiones Miller clase I-II, se ha demostrado equivalencia ${ }^{(27,28)}$. Sin embargo, es importante notar que en este tipo de procedimientos el grosor del colgajo deber ser $\geq 0.5 \mathrm{~mm}$ en el área del defecto, ya que esto mejora la predictibilidad del resultado en términos de recubrimiento radicular. Así lo reporta también Harris ${ }^{(29)}$, quien observó un recubrimiento del $26.7 \%$ en colgajos delgados contra un $95.9 \%$ en gruesos.

\section{Matriz de Colágeno Bicapa de Origen Porcino (Mucograft巴) (MG)}

Recientemente se introdujo ${ }^{(30)}$ una matriz de colágeno bilaminar de origen porcino, dicha matriz consiste en dos capas funcionales: una capa oclusiva de células que está formada en un paquete compacto de fibras colágenas y una capa porosa. Sin embargo, la capa porosa es más gruesa con el propósito de mejorar el tejido queratinizado y así inducir el efecto de crear espacio para favorecer la formación del coágulo sanguíneo. En un estudio clínico prospectivo randomizado ${ }^{(31)}$, se comparó el MG con el injerto conectivo libre, para el aumento del ancho de encía queratinizada en pacientes con prótesis parcial fija. A los 6 meses los resultados demostraron que no hubo diferencias significativas en ninguno de los dos grupos estudiados en cuanto al aumento de encía queratinizada. Sin embargo, el grupo tratado con el MG tuvo menor riesgo de morbilidad y

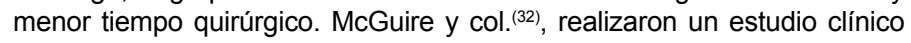
randomizado a boca partida en 25 sujetos con recesiones clase I de Miller, donde compararon el CDC + MG (test) y CDC + ITCS (control) para el tratamiento de recesiones clase. Se midieron los siguientes parámetros a los 6 meses y a un año: el recubrimiento radicular, ancho de tejido queratinizado, valores de dolor, confort y satisfacción estética. Los resultados a los 6 meses la ganancia en recubrimiento radicular entre el grupo control y test fue de $0.4 \mathrm{~mm}$, siendo mayor en el grupo control. Al año, el porcentaje de recubrimiento radicular también fue mayor en el grupo control (99.3\%) versus grupo test (88.5\%). Cuando se equilibra con los valores estéticos notificados por los pacientes y se compararon con los resultados de recubrimiento radicular históricos reportados por otros investigadores, MG + CDC presenta una alternativa viable a la ITCS + $\mathrm{CDC}$, sin la morbilidad del sitio donante de injerto de tejido blando.

\section{MOLÉCULAS BIOACTIVAS}

\section{Derivados de Matriz de Proteínas del Esmalte (DMPE)}

Los derivados de la matriz de proteínas adamantinas (DMPE) han demostrado su capacidad para promover regeneración tisular en situaciones experimentales simulando dehiscencias radiculares ${ }^{(33,34)}$ y en combinación con CDC para el tratamiento de lesiones de recesión gingival(35,36).

En un estudio comparativo prospectivo randomizado entre la utilización de CDC y la adición de DMPE a esta técnica para el recubrimiento radicular en lesiones de recesión gingival, Castellanos y col. ${ }^{(37)}$ trataron a 22 pacientes quienes exhibían lesiones Miller clase I y II. A los 12 meses de seguimiento, se encontró que las dos modalidades de tratamiento son efectivas en recubrimiento radicular. La adición de DMPE generó un recubrimiento promedio de $88.6 \%$ en comparación con un $62.2 \%$ cuando DMPE no fue adicionado. Este estudio corroboró una tendencia ya reportada por Cueva y col. ${ }^{(38)}$ unos años atrás en una investigación en 17 pacientes, tratando cincuenta y ocho defectos contralaterales de recesiones gingivales.

La estabilidad de esta terapia a corto plazo fue ilustrada en un estudio en el que 30 pacientes fueron tratados con las dos modalidades anteriormente descritas: CDC con y sin DMPE. Luego de veinticuatro meses, un $47 \%$ de las lesiones tratadas sin adición de DMPE mostraron deterioro en recubrimiento radicular en comparación con un $22 \%$ de áreas tratadas con DMPE ${ }^{(39)}$.

Siendo conscientes de los resultados obtenidos con la utilización de ITCS, Nemcovsky y col. ${ }^{(40)}$ estudiaron la eficacia clínica del
CDC con el uso adicional de DMPE para el tratamiento de las recesiones gingivales y compararlo a los ITCS. Este estudio multicéntrico en el que se trataron 70 pacientes consecutivamente ( 30 con DMPE y 40 con ITCS) con seguimiento por un periodo de 12 meses, demostró una diferencia estadísticamente significativa entre ambos grupos. Al finalizar el periodo de seguimiento, el porcentaje de recubrimiento radicular utilizando DMPE fue de $71.7 \% \pm 16.14 \%$ y de $87.0 \% \pm 12.22 \%$ con ITCS. Los autores concluyeron que aunque la utilización de DMPE es simple, predecible y menos invasivo, el recubrimiento radicular es mayor cuando se emplea ITCS. Es importante resaltar que no sólo los materiales utilizados son totalmente responsables por los resultados obtenidos. Hay otros factores como el grado de recesión inicial y el grosor del tejido perteneciente al colgajo que pueden afectar el grado de recubrimiento radicular ${ }^{(41)}$.

La formación de novo de los tres elementos esenciales para confirmar regeneración tisular (cemento, tejido conectivo y hueso) es la meta máxima y ha sido demostrada utilizando ITCS en combinación con DMPE durante el tratamiento de recesión gingival ${ }^{(42)}$. McGuire y Cochran presentaron evidencia histológica en humanos de formación nueva de cemento con fibras de ligamento periodontal organizadas e islas de hueso como resultado de la utilización de CDC en combinación con DMPE ${ }^{(43)}$. Previamente, Carnio y col. ${ }^{(44)}$ habían reportado resultados similares enfatizando que la cicatrización estaba caracterizada por la ausencia de epitelio largo de unión. Por el contrario, se observó que al combinar tejido conectivo con DMPA, este se adhería a la superficie radicular sin llevar acabo un inserción íntima al cemento.

\section{Péptidos de Adhesión Celular}

Otro de los materiales que han sido investigados en terapia mucogingival para recubrimiento radicular, es el péptido de adhesión celular $(P-15 \otimes)$ combinado con matriz inorgánica bovina derivada de hidroxiapatita (MABDH/P-15). Qian y Bhatnagar ${ }^{(45)}$ desarrollaron un material compuesto principalmente por una matriz mineral microporosa derivada de hueso bovino en combinación con un clon sintético de una cadena linear de polipéptidos pertenecientes a un complejo secuencial de 15 aminoácidos idéntica a aquella encontrada en colágeno tipo I. Esta proteína es responsable de adhesión celular, especialmente en fibroblastos y osteoblastos. Aunque este producto demostró su utilidad en procedimientos regenerativos en lesiones periodontales, en terapia de recubrimiento radicular no demostró ser eficaz. Así fue descrito por Nazareth y Cury ${ }^{(46)}$ quienes concluyeron que cuando se comparaba el CDC solo y CDC con el MABDH/P-15, no se encontró una diferencia significativa en recubrimiento radicular o ganancia de inserción clínica.

\section{Factores de Crecimiento Derivados de Plaquetas (FCDP)}

La ingeniería de tejidos tiene el potencial de facilitar el logro de resultados con técnicas de baja invasividad. Los factores de crecimiento derivados de plaquetas (FCDP) ha sido el factor de crecimiento más estudiado en periodoncia desde que se descubrió que tenía el potencial regenerativo tisular ${ }^{(47)}$.

Con base en estos descubrimientos, la versión humana recombinada de este material (hrFCDP-BB) ha sido igualmente investigada demostrando su eficacia regenerativa tanto en animales como en humanos ${ }^{(48,49)}$. En un reporte seriado de casos clínicos, McGuire y Scheyer evaluaron los resultados clínicos utilizando hrFCDP-BB con una matriz de fosfato beta tricálcico $(\beta-\mathrm{TCP})$ y una membrana de colágeno en el tratamiento de recesiones gingivales. El grupo control fue tratado con ITCS. Siete pacientes con defectos contralaterales fueron tratados y seguidos por 6 meses. Los resultados demostraron que ambos grupos no exhibieron más de $1 \mathrm{~mm}$ de recesión residual(50). Este estudio preliminar dio lugar a otra investigación en la que se examinó el potencial regenerativo de este material. Pruebas histológicas y de tomografía micro computarizada fueron utilizadas para evaluar los resultados del tratamiento de recesiones gingivales tratadas con ITCS con $0.3 \mathrm{mg} / \mathrm{ml}$ de hrFCDP-BB con una matriz de $\beta$-TCP. Los defectos clase II de Miller fueron creados quirúrgicamente y luego de 8 semanas fueron tratados de la siguiente manera: cuatro defectos recibieron terapia utilizando hrFCDPBB con una matriz de $\beta$-TCP. Los dos defectos remanentes fueron tratados con el ITCS. Ambos grupos recibieron CDC. Nueve meses después, se obtuvieron secciones que demostraron anclaje perpendicular de fibras de tejido conectivo (Fibras Sharpey) en cemento nuevo al igual que se detectó hueso formado de novo. En los especímenes tratados solamente con ITCS, no hubo evidencia de procesos regenerativos ${ }^{(51)}$. Aunque los defectos fueron creados artificialmente, los resultados de este estudio 
son estimulantes y permiten ver el potencial de agentes biológicos como una alternativa viable en terapia de defectos mucogingivales.

Membrana de Matriz Extracelular Derivada de Submucosa del Tejido del Intestino Delgado de Origen Porcino

Dynamatrix ${ }^{\circledR}$ (DM) ha ganado amplia aceptación en la reconstrucción de defectos en varias áreas del organismo. Hay numerosos reportes de su uso en paredes de hernias abdominales e inguinales, áreas ginecológicas, urológicas, paredes torácicas, modelos animales arteriales, venosos, injertos vasculares, tendones y ligamentos ${ }^{(52,53,54)}$. El uso de matrices acelulares que contienen factores de crecimiento tienen ventajas en términos de simplicidad y costo sobre las terapias de factores de crecimiento recombinadas y purificadas. Los glicosaminglicanos (GAGs) son componentes de la matriz extracelular, estos tienen importantes funciones biológicas y podrían provocar la inducción en el tipo de cicatrización ${ }^{(52)}$. La DM es una matriz de la membrana extracelular que se obtiene de la submucosa del intestino delgado de los cerdos. Mediante un proceso que conserva la composición natural de las moléculas de la matriz la DM retiene: colágeno (tipos I, III, IV y VI), glicosaminglicanos, glicoproteínas, proteoglicanos, ácido hialurónico, fibronectina, factor de crecimiento del fibroblasto, factor B de crecimiento transformante y el factor de crecimiento del fibroblasto endógeno (FCDF). Este ultimo juega un rol primordial en la cicatrización y la revascularización en la base de la herida ${ }^{(52)}$. Nevins y col.(55) compararon clínicamente e histológicamente la DM con el IGL en el aumento del ancho del tejido queratinizado y concluyeron que la DM puede ser una alternativa viable al IGL, para aumentar la dimensión del tejido queratinizado. El uso de la membrana DM puede proporcionar una fuente ilimitada de tejido del donante, lo que reduce los problemas para el clínico y la morbilidad para el paciente (Figuras 2 y 3 ).

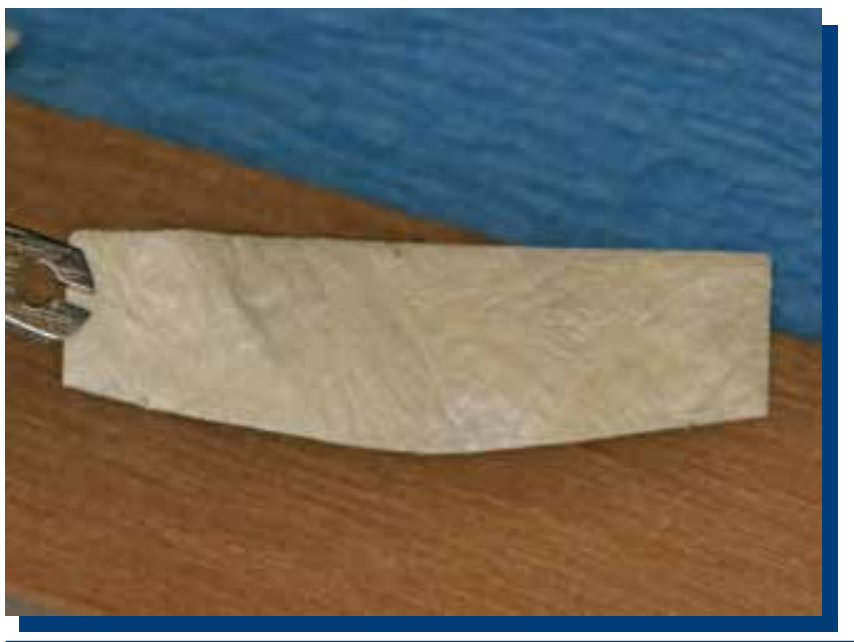

Figura 2. Membrana de matriz extracelular derivada de submucosa del tejido de intestino delgado de origen porcino (Dynamatrix) $®$ recortada y lista para colocación en lecho quirúrgico.

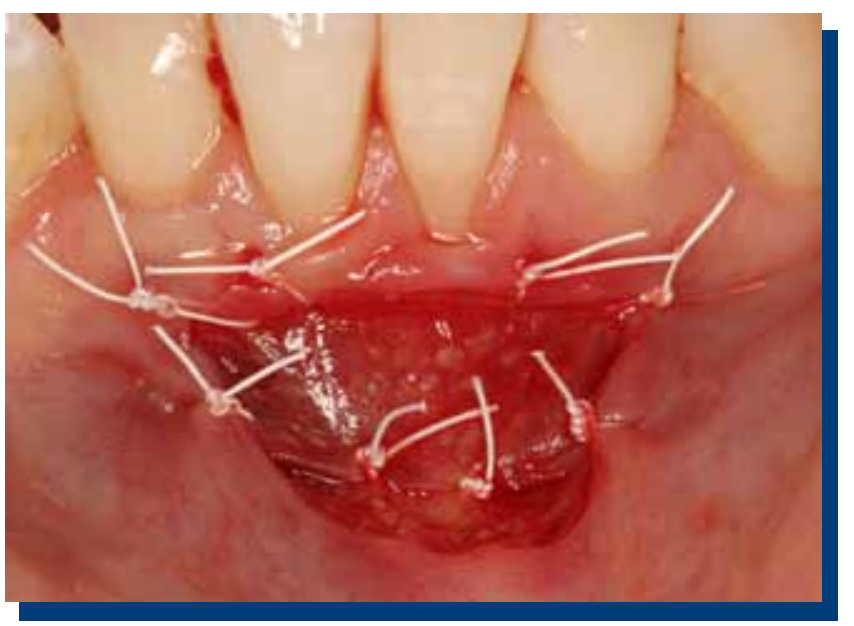

Figura 3. Membrana de matriz extracelular derivada de submucosa del tejido de intestino delgado de origen porcino (Dynamatrix) ${ }^{\circledR}$ suturada en lecho quirúrgico.
DISPOSITIVOS CON BASE EN CÉLULAS VIVAS (INGENIERÍA DE TEJIDOS)

Últimamente se han producido avances en cuanto al campo de células humanas, particularmente queratinocitos en cultivo (Graftskin o equivalente de piel humana), en el tratamiento de úlceras venosas en pacientes diabéticos ${ }^{(56,57)}$ con resultados muy favorables en la cicatrización de estas lesiones. Estos tejidos se han estudiado en relación al aumento de encía queratinizada, al recubrimiento de recesiones gingivales y al aumento de la papila proximal. Las alternativas actuales están basadas en sustitutos derivados de fibroblastos humanos autólogos y plasma rico en plaquetas.

\section{Sustitutos Derivados de Fibroblastos Humanos}

La gran ventaja de la utilización de estos injertos es que una pequeña cantidad de tejido tomado puede servir como fuente para una cantidad adecuada de material de injerto según la necesidad del paciente.

En el 2000, Pini Prato y col. ${ }^{(58,59)}$ reportó varios casos de aumento de encía con implantación de fibroblastos autólogos. El cultivo lo hacía sobre una membrana tridimensional de hidroxiapatita cuya matriz era ester benzil de ácido hialurónico. En un periodo de 15 días tenía un injerto que suturaba sobre un lecho perióstico.El aspecto inicial de cicatrización se describe como de granulación y la membrana no se detectaba a los 30 días. En todos los casos que mostró, se logró un aumento de encía queratinizada, con excelentes resultados estéticos y con un procedimiento indoloro para el paciente. Histológicamente, se obtuvo epitelio queratinizado soportado por un denso tejido conectivo después de 3 meses.

Mc Guire y Nunn ${ }^{(60)}$ hicieron la primera comparación de un sustituto dérmico derivado de fibroblasto vivo humano ( SDD- HFV) (test) con IGL(control) en el aumento de encía queratinizada en 25 pacientes con insuficiente encía adherida. El objetivo era evaluar la seguridad y efectividad de este sustituto. Los resultados son similares en la zona de control y test en cuanto recubrimiento y nivel de inserción clínica. La zona tratada con injerto libre presentó un promedio de 1 a $1.2 \mathrm{~mm}$ más de encía queratinizada a través del tiempo, y al final menor contracción. En cambio, la zona con SDD-HFV demostró tener un mejor color, así como la textura que fue significativamente mejor. Histológicamente, hubo algunas diferencias: el tejido conectivo parecía estar más organizado con SDD-HFV.

Como complemento al anterior trabajo, Mc Guire y col. ${ }^{(61)}$ presentaron un segundo estudio para evaluar la seguridad y efectividad en el uso de SDD-HFV (Dermagraft $®$ ) comparado con ITCS en el recubrimiento de una recesión gingival unitaria. El otro objetivo es saber si el comportamiento de este injerto mejoraría al estar cubierto. En ambos grupos se realizó un CDC. No hubo diferencias significativas entre ambos grupos en cuanto al recubrimiento radicular y la cantidad de encía queratinizada. Igualmente se probó la viabilidad del sustituto cubriéndolo totalmente con el CDC, cubriéndolo parcialmente y dejándolo totalmente descubierto. A pesar de que se trata de un material vivo y metabólicamente activo, no tuvo la capacidad de mantenerse viable sobre las raíces dentarias a vasculares en las zonas donde quedó descubierto.

Mohammadi y col. ${ }^{(62)}$, el 2007 hicieron un estudio comparativo de aumento de encía queratinizada entre fenestración en periostio (experimental) vs injerto gingival de cultivo de fibroblastos (control). Se trabajó en 9 pacientes, en dientes contralaterales. En todos las zonas tratadas hubo un aumento de encía queratinizada. Sin embargo, ésta fue mayor en el grupo experimental $(2.8 \mathrm{~mm})$ en comparación al grupo control ( $2 \mathrm{~mm})$, siendo esta diferencia significativa.

TCB (Terapia Celular Bilaminar) es un producto de células vivas construido con colágeno tipo 1 de origen bovino (tendones y luego purificado), fibroblastos y queratinocitos aislados de prepucio humano. Se ha demostrado la presencia de numerosas citoquinas producidas por las células de la matriz TCB. Éstas incluyen factores de crecimiento relacionados con la reparación periodontal (factor de crecimiento derivado de plaqueta, proteína-7 morfogenética de hueso y factor de crecimiento vascular endotelial (VEGF) (Figura 4). 


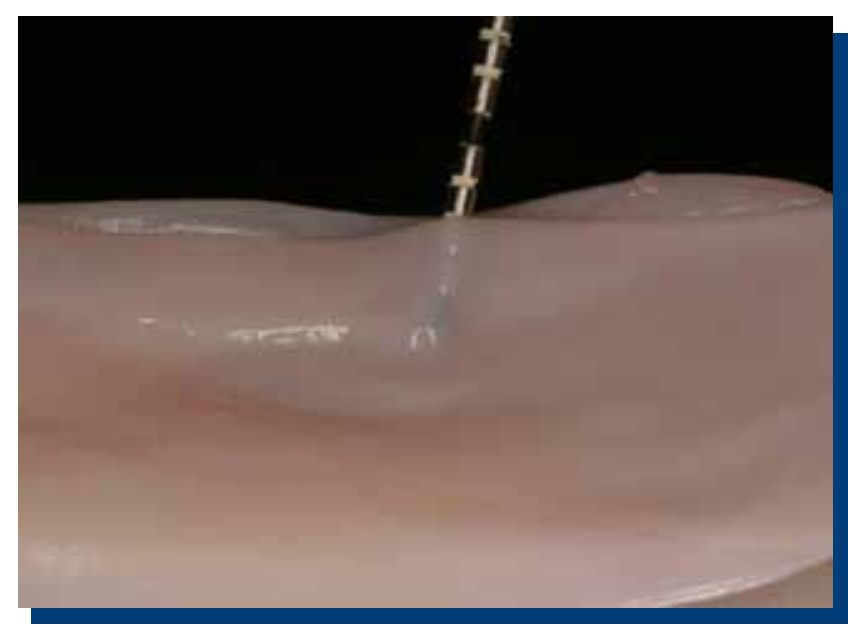

Figura 4. Matriz de células vivas (del inglés: Living cellular construct).

Nevins ${ }^{(63)}$ utilizó un TCB en 4 pacientes para aumentar encía queratinizada. La completa epitelización se obtuvo a los 14 días. El color y aspecto eran similares a los tejidos vecinos. Entre las 3 y 7 semanas postquirúrgicas realizó estudios histológicos de los sitios tratados y análisis de ADN de estas muestras. Estas biopsias mostraron zonas en reparación con aspecto normal gingival y presencia de epitelio paraqueratinizado. El análisis de ADN, demostró que sólo el ADN del paciente se encontraba presente al momento de la biopsia.

Mc Guire y col.(64) evaluaron la seguridad y efectividad en el uso de un producto compuesto por fibroblastos y queratinocitos de tejido neonatal viable (TCB) comparado con IGL en aumento de encía queratinizada sin necesidad de recubrimiento radicular. Se estudiaron 25 sujetos a los que se les hizo las dos técnicas (TCB e IGL) en zonas que tenían escasa encía insertada. Los resultados demostraron que TCB es seguro y capaz de generar encía queratinizada. Con IGL se creó más encía queratinizada. Sin embargo, 24 de los 25 sitios tratados con TCB demostraron un aumento en encía queratinizada después de 6 meses. TCB es similar en las otras mediciones a IGL (nivel de inserción clínica, recesión, inflamación y resistencia a la tracción muscular). TCB tiene mejor resultado en color y textura y percepción de dolor y sensibilidad.

Recientemente ${ }^{(65)}$, se ha estudiado los marcadores angiogénicos durante la primera etapa de la cicatrización usando un TCB y comparándolo con IGL. Éstos cicatrizan de diferentes maneras ya que el IGL repara por primera intención y el TCB, inversamente, no se integraría a los tejidos que la rodean, pero si modularía la cicatrización del tejido de base y adyacente. Se ha postulado que mejora el entorno de la herida a través de la interacción del factor de crecimiento, la deposición y degradación de la matriz, el recubrimiento de la herida, y el suministro de células de respuesta. Parece ser que estimula la propia respuesta celular para regenerar un tejido apropiado in situ a través del cual se produce un la modulación y desarrollo de la cicatrización por segunda intención. Esto aún no se ha aclarado.

Mc Guire y col.(66) realizaron un estudio en 96 pacientes, los cuales recibieron en el mismo día tratamiento con IGL y en el lado contralateral Matriz Celular Viva (MCV-también denominado TCB). Se realizaron controles a la semana, 4 semanas, 3 meses y 6 meses. A los 6 meses, MCV regeneró mayor o igual a $2 \mathrm{~mm}$ en el $95.3 \%$ de los pacientes. El IGL generó más encía queratinizada que $\mathrm{MCV}$, pero la encía era de color y textura similar a la encía adyacente con este último. Murata y col. ${ }^{(67)}$, trataron14 sitios en 4 pacientes utilizando un sustituto dérmico compuesto por fibroblastos gingivales tomados de la zona retro mandibular y cultivados en una matriz. Después de 3 meses, se trataron las recesiones tipo I y II de Miller, colocando sobre el defecto la membrana y cubriéndolo con un CDC.

A pesar de que este estudio no ocupó grupo de control, los resultados mostraron un desarrollo clínico favorable, logrando no sólo recubrimiento radicular de un $75.2 \%$ a las 30 semanas, si no que también aumento de encía queratinizada y encía adherida.

Mc Guire y Scheyer ${ }^{(68)}$ hicieron un estudio para evaluar la eficacia de inyectar fibroblastos autólogos en la zona de papila interproximal. A pesar de que el análisis estadístico indica que el grupo test fue superior al placebo, existieron fallas en relación a poder mostrar la evidencia del tratamiento en relación a la distancia desde la cima de la papila a la base del área de contacto al medirla con una sonda periodontal, en el ancho de la papila y la distancia desde la cima papilar o la base del área de contacto hacia la cresta ósea. Los resultados obtenidos sugieren que podría ser efectivo y seguro para el tratamiento de la insuficiencia papilar.

\section{Plasma Rico en Plaquetas}

Cheung y Griffin $^{(69)}$ revisan la eficacia de un injerto con concentrado de plaqueta versus ITCS en tratamiento de recesiones gingivales de tipo I y II de Miller. Se trataron 17 arcos en 15 pacientes con CDC, obteniendo el $80 \%$ de recubrimiento con concentrado de plaquetas y $95 \%$ con injerto conectivo a los 8 meses. El mejor aspecto estético se logró con concentrado de plaqueta. En el estudio de Huang y col..$^{(70)}$, se trataron recesiones gingivales unitarias tipo I de Miller en 24 pacientes comparando el CDC y CDC + PRP. Los resultados se observaron a las $2,4,12$ y 24 semanas. No se logró demostrar que la aplicación de PRP en CDC proporcione mejoras clínicamente apreciable en los resultados finales de la terapéutica. Ferreira y col. ${ }^{(71)}$ realizaron un estudio para analizar histométricamente el proceso de cicatrización de una recesión tratada con ITCS + PRP vs ITCS, en 6 perros. Se encontró la formación de una mayor longitud de nuevo cemento en los sitios tratados con PRP. A su vez, Aroca y col. ${ }^{(72)}$ analizaron si la utilización de una membrana de plaquetas rica en fibrina autóloga mejora los parámetros clínicos CDCT en el tratamiento de recesiones múltiples Miller clase I o II. EI CDCT es un tratamiento predecible en el recubrimiento de defectos clase I o II de Miller $(91.5 \%)$ y la colocación de una membrana de plaqueta debajo del colgajo produjo menos recubrimiento $(80.7 \%)$ a los 6 meses. En cambio, en cuanto a la cantidad de encía queratinizada, se logró un aumento estadísticamente significativo en las zonas que se adicionó PRP.

\section{DISCUSIÓN}

La realidad clínica del carácter invasivo de los procedimientos que utilizan tejidos autográficos y la falta de una cantidad adecuada de tejidos del área donante son afrontadas diariamente en el tratamiento de lesiones mucogingivales. El riesgo de una intervención quirúrgica prolongada ha sido previamente demostrado por Griffin y col.(3), quienes compararon la frecuencia de complicaciones postoperatorias entre los diferentes procedimientos de injerto de tejido blando. Por cada minuto del procedimiento se produjo un aumento del $4 \%$ en la probabilidad de desarrollar dolor de moderado a severo y un aumento del $3 \%$ de moderada o severa inflamación. Por estas y otras razones, la viabilidad de la utilización de sustitutos tisulares para los pacientes con defectos mucogingivales se está convirtiendo en una realidad y una alternativa promisoria.

Al analizar los aloinjertos, en los estudios de corto plazo para el tratamiento de recubrimiento radicular (menos de 12 meses) ${ }^{(13,14,15)}$, se observa una similitud estadística entre la MDA vs ITCS, y la MDA vs CDC. Harris ${ }^{(16)}$ demostró que a los 4 años, existe una superioridad estadística de ITCS en comparación a MDA, lo que nos hace pensar que el comportamiento a largo plazo de la MDA es su punto débil.

Por otra parte, para el tratamiento del aumento de encía queratinizada, existe una tendencia de mayor formación de tejido queratinizado con el ITCS en comparación con MDA ${ }^{(7,8)}$. La misma tendencia se observa pero en menor tamaño entre MDA y IGL ${ }^{(1)}$. Esto se podría explicar porque la MDA es un trasplante de un injerto no vital procedente de un individuo genéticamente diferente en su epitelio, y éste puede carecer de la capacidad inherente a la cito diferenciación directa de la superficie del epitelio oral nativo.

Los estudios con fibroblastos humanos pretenden aumentar cantidad de encía queratinizada ${ }^{(5,59,60,62)}$. Está claro que es posible generar encía queratinizada con estos materiales, pero no en gran cantidad ${ }^{(60)}$. Al comparar estos sustitutos con $\operatorname{IGL}^{\left({ }^{(60,65)}\right.}$ el tejido logrado tiene un aspecto más estético. Faltan más estudios clínicos controlados. En cuanto al $\mathrm{TCB}^{(63,65)}$, es una alternativa viable y segura de generación de encía queratinizada, con resultados similares a los estudios anteriores. Será interesante aclarar la respuesta biológica del tejido, ya que el TCB "se disipa" de la curación del sitio. Al parecer actúa como un agente de curación de heridas, en lugar de un injerto. Más estudios clínicos e histológicos son necesarios para evaluar la utilidad del TCB en la práctica clínica. 
Al ser usados para lograr recubrimiento radicular(61,64), el SDD$\mathrm{FH}$ ofrece ser un potencial material para recubrir recesiones tipo I y II de Miller.

El uso de PRP demostró poder mejorar la cicatrización de los tejidos blandos, promoviendo la estabilización inicial de los colgajos e injertos en el recubrimiento radicular ${ }^{(69)}$. Sin embargo, esto no pudo demostrarse en otros estudios ${ }^{(70,71)}$, y el adicionar PRP no proporcionó mejoras clínicamente apreciables en los resultados finales de la terapéutica lo que no justificaría el uso de PRP para el tratamiento en los defectos tipo I o II de Miller.

Al seleccionar el material que va a ser utilizado para procedimientos de cirugía plástica periodontal, se debe tener en cuenta que estos materiales ofrezcan un resultado predecible, que permitan la regeneración tisular a través del seguimiento de principios biológicos de cicatrización y minimizar el trauma para los pacientes. El advenimiento de biomateriales de base biológica, ingeniería de tejidos y moléculas bioactivas presentan una alternativa promisoria en comparación con procedimientos tradicionales. Este nuevo grupo de materiales quirúrgicos están aun en su infancia y no se cuenta con el respaldo científico longitudinal que permita definir con certeza su capacidad y la consistencia de su desempeño al largo plazo ${ }^{(73)}$. Su utilización puede incrementar el potencial regenerativo de tejidos periodontales y reducir trauma y dolor postquirúrgico en los pacientes ${ }^{(74)}$. La falta de evidencia de carácter consistente en cuanto a los beneficios adjuntos de terapias combinadas junto con un análisis de costo-beneficio debe ser considerado en el momento de escoger el tipo de material y de procedimiento a efectuarse.

En conclusión, los resultados de la revisión son los siguientes:

- Los ITCS, CDC solos o asociado con injertos o biomateriales MDA, DMPE y ITCS y la regeneración tisular guiada (RTG) con membranas de colágeno pueden usarse como procedimientos de recubrimiento radicular.

- En los casos en que se espera recubrimiento radicular y aumento en el ancho del tejido queratinizado, el uso de ITCS y IGL respectivamente, parecen ser los tratamientos más predecibles.

- La MDA pueden ser una opción en los casos donde los ITCS tomados del paladar no son suficientes para cubrir un área de recesión.

- La literatura disponible relacionada con DMPE ha demostrado que aunque en teoría ofrece un potencial regenerativo y de aceleración del proceso de cicatrización, cuando es llevado a la práctica, la utilización de estas moléculas biológicas no parece llenar estas expectativas y ha sido reportado consistemente que su recubrimiento radicular es inferior al obtenido utilizando ITCS.

- La evidencia de recubrimiento radicular utilizando péptidos de adhesión celular es escasa y se requiere de más estudios comparativos para poder establecer criterios de indicaciones y aplicaciones de este materia en este campo.

- El recubrimiento radicular utilizando los FCDP, aunque en su infancia, están respaldados por evidencia histológica humana que permite sugerir que este material tiene la capacidad de soportar no solo recubrimiento radicular sino regeneración de tejidos de manera simultanea. Estudios independientes que corroboren esta información son necesarios para poder confirmar los hallazgos preliminares recién publicados.

- El potencial de cicatrización utilizando membranas bioactivas como DM es promisorio. Aunque se cuenta con un respaldo numeroso en la literatura médica, en el campo de cirugía mucogingival los estudios disponibles son escasos. Su comportamiento a largo plazo, está aun por verificarse y se necesitan estudios independientes y con seguimiento de por lo menos cinco años para poder validarlos y entrar a recomendar los beneficios de este tipo de terapia.

- La evidencia disponible es limitada verificando el beneficio del PRP en el recubrimiento radicular y los investigadores recomiendan futuros estudios en los cuales se incluya un mayor número de muestras y tiempo de evaluación más largo.

- En cuanto al TCB (MCV), los resultados positivos obtenidos, sugieren que pueden ser una alternativa segura y viable en la restauración de tejido queratinizado cuando el recubrimiento radicular no es necesario.

- También sigue siendo difícil especular cómo la inclusión de más estudios ramdomizados y con seguimientos a más largo plazo podrían afectar los resultados. Faltan aun estudios que consideren ampliarse e estándar de 6 a 12 meses realizado en el cirugía plástica periodontal.

\section{CONFLICTOS DE INTERÉS}

Los autores declaran no tener conflictos de interés.

\section{REFERENCIAS BIBLIOGRÁFICAS}

1. Wei $P$, Laurell $L$, Geivelis $M$, Lingen $M$. Acellular dermal matrix allografts to achieve increased attached gingiva. Part 1. A clinical study. J Periodontol, 2000; 71: $1297-1305$

2. Langer B, Langer L. Subepithelial connective tissue graft technique for root coverage. J Periodontol, 1985; 56: 715-720.

3. Griffin TJ, Cheung WS, Zavras AI, Damoulis PD. Postoperative complications following gingival augmentation procedures. J Periodontol, 2006; 77: 2070-2079.

4. Shulman J. Clinical evaluation of an acellular dermal allograft for increasing the zone attached gingiva. Pract Periodontics Aesthetic Dent, 1996; 8: 201-208.

5. Gapski R, Parks C, Wang H. Acellular dermal matrix for mucogingival surgery: A meta-analysis. J Periodontol, 2005; 76: 1814-1822.

6. Harris R. Clinical evaluation of 3 techniques to augment keratinized tissue without root coverage. J Periodontol, 2001; 72: 932-938.

7. Scarano A, Barros R, lezzi G, Piattelli A, Novaes Jr. A. Acellular dermal matrix graft for gingival augmentation: A preliminary clinical, histologic, and ultrastructural evaluation. J Periodontol, 2009; 80: 253-259.

8. Wei P, Laurell L, Geivelis M, Lingen M, Maddalozzo D. Acellular dermal matrix allografts to achieve increased attached gingiva. Part 2. A histological comparative study. J Periodontol, 2002; 73: 257-265.

9. Richardson R, Maynard G. Acellular dermal graft: A human histologic case report. Int J Periodontics Restorative Dent, 2002; 22: 21-29.

10. Cummings L, Kaldahl W, Allen EP. Histologic evaluation of autogenous connective tissue and acellular dermal matrix grafts in humans. J Periodontol, 2005 76: 178-186.

11. Luczyszyn S, Grisi M, Novaes A, Palioto D, Souza S, Taba M. Histologic analysis of the acellular dermal matrix graft incorporation process: A pilot study in dogs. Int $J$ Periodontics Restorative Dent, 2007; 27: 341-347.

12. Aichelmann-Reidy ME, Yukna RA, Evans GH et al. Clinical evaluation of acellular allograft dermis for the treatment of human gingival recession. J Periodontol, 2001; 72: 998-1005.
13. Novaes AB Jr, Grisi DC, Molina GO et al. Comparative 6-month clinical study of a subepithelial connective tissue graft and acellular dermal matrix graft for the treatment of gingival recession. J Periodontol, 2001; 72: 1477-1484.

14. Tal $H$, Moses $O$, Zohar $R$ et al. Root coverage of advanced gingival recession: A comparative study between acellular dermal matrix allograft and subepithelial connective tissue grafts. J Periodontol, 2002; 73: 1405-1411.

15. Harris R. A short-term and long-term comparison of root coverage with an acellular dermal matrix and subepithelial graft. J Periodontol, 2004; 75: 734-743.

16. Hirsch A, Goldstein M, Goultschin J, Boyan BD, Schwartz Z. A 2-year follow-up of root coverage using subpedicle acellular dermal matrix allografts and subepithelial connective tissue autografts. J Periodontol, 2005; 76: 1323-1328.

17. Woodyard JG, Greenwell H, Hill M et al. The clinical effect of acellular dermal matrix on gingival thickness and root coverage compared to coronally positioned flap alone. J Periodontol, 2004; 75: 44-56.

18. De Queiroz Cortez A, Martins A, Nociti F, Sallum A, Casati M, Sallum E. Coronally positioned flap with or without acellular dermal matrix graft in the treatments of class i gingival recessions: A randomized controlled clinical study. J Periodontol, 2004; 75: $1137-1144$

19. Barros RM, Novaes AB Jr, Grisi MFM et al. A 6-month comparative clinical study of a conventional and a new surgical approach for root coverage with acellular dermal matrix. J Periodontol, 2004; 75: 1350-1356.

20. Taylor J, Gerlach R, Herold R, Bisch F, Dixon D. A modified tensionless gingival grafting technique using acellular dermal matrix. Int $J$ Periodontics Restorative Dent, 2010; 30: 513-521.

21. Felipe M, Andrade P, Grisi M, Souza S, Taba Jr. M, Palioto D, Novaes Jr. A Comparison of two surgical procedures for use of the acellular dermal matrix graft in the treatment of gingival recessions: A randomized controlled clinical study. Periodontol, 2007; 78: 1209-1217.

22. Papageorgakopoulos G, Greenwell H, Hill M, Vidal R, Scheetz J. Root coverage using acellular dermal matrix and comparing a coronally positioned tunnel to a 
coronally positioned flap approach. J Periodontol, 2008; 79: 1022-1030. 23. Modarressi $\mathrm{M}$, Wang $\mathrm{H}$. Tunneling procedure for root coverage using acellular dermal matrix: A case series. Int J Periodontics Restorative Dent, 2009; 29: 395403

24. Mahn D. Use of the tunnel technique and an acellular dermal matrix in the treatment of multiple adjacent teeth with gingival recession in the esthetic zone. Int $J$ Periodontics Restorative Dent, 2010; 30: 593-599.

25. Shepherd N, Greenwell H, Hill M, Vidal R, Scheetz J. Root coverage using acellular dermal matrix and comparing a coronally positioned tunnel with and without platelet-rich plasma: A pilot study in humans. J Periodontol, 2009; 80: 397-404

26. Bunyaratavej P, Wang H. Collagen membranes: A review. J Periodontol, 2001; 72: $215-229$

27. Cetiner D, Parlar A, Balos K, Alpar R. Comparative clinical study of connective tissue graft and two types of bioabsorbables barries in the treatment of localized gingival recessions. J Periodontol, 2003; 74: 1196-1205.

28. Cardaropoli $D$, Cardaropoli $D$. Healing of gingival recessions using a collagen membrane with demineralized xenograft: A ramdomized controlled clinical trial. Int $J$ Periodontics Restorative Dent, 2009; 29: 59-68.

29. Harris R. A comparative study of root coverage obtained with guide tissue regeneration utilizing a bioabsorbable membrane versus the connective tissue with parcial-thickness double pedicle graft. J Periodontol, 1997; 68: 769-790.

30. Herford A, Akin L, Cicciu M, Maiorana C Boyne. Use of a porcine collagen matrix as an alternative to autogenous tissue for grafting oral soft tissue defects. $J$ Oral Maxillofac Surg, 2010; 68: 1463-1470.

31. Sanz M, Lorenzo R, Aranda JJ, Martin C, Orsini M. Clinical evaluation of a new collagen matrix (Mucografts prototype) to enhance the width of keratinized tissue in patients with fixed prosthetic restorations: A randomized prospective clinical trial. $J$ Clin Periodontol, 2009; 36: 868-876.

32. McGuire M, Scheyer T. Xenogeneic collagen matrix with coronally advanced flap compared to connective tissue with coronally advanced flap for the treatment of dehiscence-type recession defects Michael K. J Periodontol, 2010; 81: 1108-1117. 33. Heijl L. Periodontal regeneration with enamel matrix derivative in one human experimental defect. A case report. J Clin Periodontol, 1997; 24: 693-696.

34. Hammastrom L, Heijl L, Gestrelius S. Periodontal regeneration in a buccal dehiscence model in monkeys after applications of enamel matrix proteins. J Clin Periodontol, 1997; 24: 669-677.

35. Andrade PF, Grisi MFM, Marcaccini AM, Fernades PG, Reino DM, Souza SLS et al. Comparison between micro and macrosurgical techniques for the treatment of localized gingival recessions using coronally positioned flaps and enamel matrix derivative. J Periodontol, 2010; 81: 1572-1579.

36. Pilloni A, Paolantonio M, Camargo PM. Root coverage with a coronally positioned flap used in combination with enamel matrix derivative: 18-month clinical evaluation. J Periodontol, 2006; 77: 2031-2039.

37. Castellanos A, de la Rosa M, de la Garza M, Caffesse RG. Enamel matrix derivative and coronal flaps to cover marginal tissue recessions. J Periodontol, 2006; 77: 7-14

38. Cueva MA, Boltchi FE, Hallmon WW, Nunn ME, Rivera-Hidalgo F, Rees T. A comparative sudy of coronally advancd flaps with and without the addition of ename matrix derivative in the treatment of marginal tissue recession. $J$ Periodontol, 2004; 75: $949-956$

39. Spahr A, Haegewald S, Tsoulfidou F, Rompola E, Heijl L, Bernimoulin JP et al Coverage of Miller class I and II recession defects using enamel matrix proteins versus coronally advanced flap technique: A 2 year report. J Periodontol, 2005; 76: 1871-1880.

40. Nemcovsky CE, Artzi Zvi, Tal H, Kozlovsky A, Moses O. A multicenter comparative study of two root coverage procedures: Coronally advanced flap with addition of enamel matrix proteins and subpedicle connective tissue graft. $J$ Periodontol, 2004; 75: 600-607.

41. Berlucchi I, Francetti L, Del Fabbro M, Basso M, Weinstein RL. The influence of anatomical features on the outcome of gingival recessions treated with coronally advanced flap and enamel matrix derivative: A 1 year prospective study. J Periodontol, 2005; 76: 899-907.

42. Rasperini G, Silvestri M, Schenk RK, Nevins ML. Clinical and histologic evaluation of human gingival recession treated with a subepithelial connective tissue graft and enamel matrix derivative (Emdogain): A case report. Int J Periodontics Restorative Dent, 2000; 20: 269-275.

43. McGuire MK, Cochran DL. Evaluation of human recession defects treated with coronally advanced flaps and either enamel matrix derivative or connective tissue. Part 2: Histological evaluation. J Periodontol, 2003; 74: 1126-1135.

44. Carnio J, Camrgo PM, Kenney EB, Schenk RK. Histological evaluation of 4 cases of root coverage following a connective tissue graft combined with an ename matrix derivative preparation. J Periodontol, 2002; 73: 1534-1543.

45. Qian JJ, Bhatnagar RS. Enhanced cell attachment to anorganic bone mineral in the presence of a synthetic peptide related to collagen. J Biomed Mater Res, 1996; 3: $545-554$

46. Nazareth CA, Cury PR. Use of anorganic bovine-derived hydroxyapatite matrix $(A B M) / c e l l-b i n d i n g ~ p e p t i d e(p-15)$ in the treatment of isolated class I gingival recession defects: A pilot study. J Periodontol, 2011; 82: 700-707.

47. Lynch SE, Williams RC, Polson AM et al. A combination of platelet-derived growth factor and insulin-like growth factor enhances periodontal regeneration. $J$ Clin Periodontol, 1989; 16: 545-554.

48. Matsuda N, Lin WI, Kumar MI, Cho MI, Genco RJ. Mitogenic, chemotactic and synthetic responses of rat periodontal ligament fibroblastic cells to polypeptide growth factors in vitro. J Periodontol, 1992; 63: 515-525.

49. Nevins M, Camelo M, Nevins ML, Schenk RK, Lynch SE. Periodontal regeneration in humans using recombinant human platelet-derived growth factorBB (rhPDGF-BB) and allogenic bone. J Periodontol, 2003; 74: 1282-1292.
50. McGuire MK, Scheyer ET. Comparison of recombinant human platelet derived growth factor BB plus beta tricalcium phosphate and a collagen membrane to subepithelial connective tissue grafting for the treatment of recession defects: A case series. Int J Periodontics Restorative Dent, 2006; 26: 127-133.

51. McGuire MK, Scheyer ET, Nevins M, Schupbach P. Evaluation of human recession defects treated with coronally advanced flaps and either purified recombinant human platelet-derived growth factor-BB with beta tricalcium phosphate or connective tissue: A histologic and microcmputed tomographic examination. Int J Periodontics Restorative Dent, 2009; 29: 7-21.

52. Hodde J, Badylak S. Glycosaminoglycan content of small intestinal submucosa: A bioscaffold fot tissue replacement. Tissue Engineering, 1996; 2: 209-216.

53. Smith M, Campbell R. Use of a biodegradable patch for reconstruction of large thoracic cage defects in growing children. J Pediatr Surg, 2006; 41: 46-49.

54. Woo SL, Takakura Y, Liang R, Jia F, Moon DK. Treatment with bioscaffold enhances the the fibril morphology and the collagen composition of healing medial collateral ligament in rabbits. Tissue Eng, 2006 Jan; 12(1): 159-166.

55. Nevins M, Camelo M, Schupbach P, Kim D. The clinical efficacy of dynamatrix extracellular membrane in augmenting keratinized tissue. Int $\mathrm{J}$ Periodontics Restorative Dent, 2010; 30: 151-161.

56. Falanga $\mathrm{V}$, Margolis D, Alvarez $\mathrm{O}$, Auletta M, Maggiacomo F, Altman M, Jensen J, Sabolinski M, Hardin-Young J. Rapid healing of venous ulcers and lack of clinical rejection with an allogeneic cultured human skin equivalent. Human skin equivalent investigators group. Arch Dermatol, 1998; 134: 293-300.

57. Veves A, Falanga V, Armstrong DG, Sabolinski ML. Apligraf diabetic foot ulcer study. Graftskin, a human skin equivalent, is effective in the managment of noninfected neurophatic diabetic foot ulcers. Diabetes Care, 2001; 24: 290-295.

58. Pini Prato GP, Rotundo R, Magnani C, Soranzo C. Tissue engeineering technology for gingival augmentation procedures: A case report. Int $J$ Periodontics Restorative Dent, 2000; 20: 553-559.

59. Pini Prato GP, Rotundo R, Magnani C, Soranzo C, Muzzi L, Cairo F. An autologous cell hyaluronic acid graft technique for the gingival augmentation: A case series. J Periodontol, 2003; 74: 262-267.

60. McGuire MK, Nunn ME. Evaluation of the safety and efficacy of periodonta applications of a living tissue-engineered human fibroblast-derived dermal substitute. I. Comparison to the gingival autograft: A randomized controlled pilot study. J Periodontol, 2005 Jun; 76(6): 867-880.

61. Wilson TG Jr, McGuire MK, Nunn ME. Evaluation of the safety and efficacy of periodontal applications of a living tissue-engineered human fibroblast-derived dermal substitute. II. Comparison to the subepithelial connective tissue graft: A randomized controlled feasibility study. J Periodontol, 2005 Jun; 76(6): 881-889.

62. Mohammadi M, Shokrgozar MA, Mofid R. Culture of human gingival fibroblasts on a biodegradable scaffold and evaluation of its effect on attached gingiva: A randomized, controlled pilot study. J Periodontol, 2007 Oct; 78(10): 1897-1903.

63. Nevins ML. Tissue-engineered bilayered cell therapy for the treatment of oral mucosal defects: A case series. Int J Periodontics Restorative Dent, 2010 Feb; 30(1): 31-39

64. McGuire MK, Scheyer ET, Nunn ME, Lavin PT. A pilot study to evaluate a tissueengineered bilayered cell therapy as an alternative to tissue from the palate. $J$ Periodontol, 2008 Oct; 79(10): 1847-1856.

65. Morelli T, Neiva R, Nevins ML, McGuire MK, Scheyer ET, Oh TJ, Braun TM, Nör JE, Bates D, Giannobile WV. Angiogenic biomarkers and healing of living cellular constructs. J Dent Res, 2011 Apr; 90(4): 456-462. Epub 2011 Jan 19.

66. McGuire MK, Scheyer ET, Nevins M, Neiva R, Cochran DL, Mellonig JT Giannobile WV, Bates D. Living cellular construct for increasing the width of keratinized gingiva. Results from a randomized, within-patient, controlled trial. $J$ Periodontol, 2011 Mar 29. [Epub ahead of print].

67. Murata M, Okuda K, Momose M, Kubo K, Kuroyanagi Y, Wolff LF. Root coverage with cultured gingival dermal substitute composed of gingival fibroblasts and matrix: A case series. Int J Periodontics Restorative Dent, 2008 Oct; 28(5): 461-467.

68. McGuire MK, Scheyer ET. A randomized, double-blind, placebo-controlled study to determine the safety and efficacy of cultured and expanded autologous fibroblast injections for the treatment of interdental papillary insufficiency associated with the papilla priming procedure. J Periodontol, 2007 Jan; 78(1): 4-17.

69. Cheung WS, Griffin TJ. A comparative study of root coverage with connective tissue and platelet concentrate grafts: 8-month results. J Periodontol, 2004 Dec; 75(12): 1678-1687.

70. Huang LH, Neiva RE, Soehren SE, Giannobile WV, Wang HL. The effect of platelet-rich plasma on the coronally advanced flap root coverage procedure: A pilot human trial. J Periodontol, 2005 Oct; 76(10): 1768-1777.

71. Suaid FF, Carvalho MD, Santamaria MP, Casati MZ, Nociti FH Jr, Sallum AW, Sallum EA. Platelet-rich plasma and connective tissue grafts in the treatment of gingival recessions: A histometric study in dogs. J Periodontol, 2008 May; 79(5): $888-895$

72. Aroca S, Keglevich T, Barbieri B, Gera I, Etienne D. Clinical evaluation of a modified coronally advanced flap alone or in combination with a platelet-rich fibrin membrane for the treatment of adjacent multiple gingival recessions: A 6-month study. J Periodontol, 2009 Feb; 80(2): 244-252.

73. Leong DJM, Wang HL. A decision tree for soft tissue grafting. Int $J$ Periodontics Restorative Dent, 2011; 31: 307-313.

74. Rasperini G, Roccuzzo M, Francetti L et al. Subepithelial connective tissue graft for treatment of gingival recessions with and without enamel matrix derivative: $A$ multicenter, randomized controlled clinical trial. Int J Periodontics Restorative Dent 2011; 31: 133-139. 